University of Nebraska - Lincoln

DigitalCommons@University of Nebraska - Lincoln

U.S. Environmental Protection Agency Papers

U.S. Environmental Protection Agency

2004

\title{
Analysis of functional effects of a mixture of five pesticides using a ray design
}

Chris Gennings

Solveritas, L.L.C., gennings@hsc.vcu.edu

W. Hans Carter Jr.

Solveritas, L.L.C.

Michelle Casey

Virginia Commonwealth University

Virginia Moser

Virginia Commonwealth University

Richard Carchman

Solveritas, L.L.C.

See next page for additional authors

Follow this and additional works at: https://digitalcommons.unl.edu/usepapapers

Gennings, Chris; Carter, W. Hans Jr.; Casey, Michelle; Moser, Virginia; Carchman, Richard; and Simmons, Jane Ellen, "Analysis of functional effects of a mixture of five pesticides using a ray design" (2004). U.S. Environmental Protection Agency Papers. 167.

https://digitalcommons.unl.edu/usepapapers/167

This Article is brought to you for free and open access by the U.S. Environmental Protection Agency at DigitalCommons@University of Nebraska - Lincoln. It has been accepted for inclusion in U.S. Environmental Protection Agency Papers by an authorized administrator of DigitalCommons@University of Nebraska - Lincoln. 


\section{Authors}

Chris Gennings, W. Hans Carter Jr., Michelle Casey, Virginia Moser, Richard Carchman, and Jane Ellen Simmons 


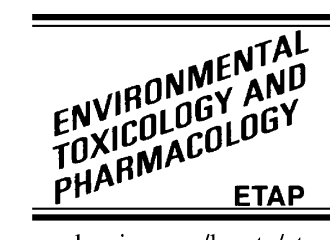

www.elsevier.com/locate/etap

\title{
Analysis of functional effects of a mixture of five pesticides using a ray design
}

\author{
Chris Gennings ${ }^{\mathrm{c}, *}$, W. Hans Carter Jr. ${ }^{\mathrm{c}}$, Michelle Casey $^{\mathrm{a}}$, Virginia Moser ${ }^{\mathrm{b}}$, \\ Richard Carchman ${ }^{\mathrm{c}}$, Jane Ellen Simmons ${ }^{\mathrm{b}}$ \\ ${ }^{a}$ Department of Biostatistics, Virginia Commonwealth University, Richmond, VA 23298-0032, USA \\ b Neurotoxicology Division, NHEERL-ORD, U.S. EPA, MD B105-04, Research Triangle Park, NC 27711, USA \\ ${ }^{\mathrm{c}}$ Solveritas, L.L.C., Richmond, VA 23219, USA \\ Received 28 April 2003; accepted 18 March 2004 \\ Available online 7 October 2004
}

\begin{abstract}
The protection of human health from the adverse effects of cumulative environmental exposure to chemical mixtures is an important issue. Of particular interest is the detection and characterization of interaction among chemicals in complex mixtures. Response surface methodology, often supported by factorial designs, is the classical statistical experimental approach. Fixed-ratio ray designs, which may include the use of single chemical data in addition to data along mixture ray(s), have been proposed as an alternative approach. Such designs permit a reduction in the amount of experimental effort when the region of interest can be restricted to exposure-relevant mixing ratios. A 'single chemicals required' (SCR) approach and a 'single chemicals not required' (SCNR) approach are both described. The methods are illustrated with a five-chemical mixture of organophosphorus pesticides-acephate (ACE), diazinon (DIA), chlorpyrifos (CPF), malathion (MAL) and dimethoate (DIM). Their relative proportions in the mixture were based on the relative dietary human exposure estimates of each chemical as projected by the U.S. EPA Dietary Exposure Evaluation Model (DEEM).

Use of the SCR and SCNR methods for binary endpoints are demonstrated using a dichotomized gait score as an indication of toxicity. For both methods, the overall hypothesis of additivity was rejected, indicating significant departure from additivity when the five pesticides were combined at the specified mixing ratio. By comparison of the predicted response under additivity to the modeled response of the experimental mixture data this departure from additivity was characterized as synergy (greater than additive toxicity). To examine the influence of malathion in the mixture, it was removed from the five-pesticide mixture (full ray) and the remaining four chemicals (reduced ray) were combined at the same relative proportions used in the full fixed-ratio ray There was not a significant departure from additivity along the ray with the four remaining pesticides omitting malathion. Thus, although malathion was not dose-responsive alone, it significantly interacted with the other pesticides.
\end{abstract}

(C) 2004 Elsevier B.V. All rights reserved.

Keywords: Additivity; Interaction; Dose addition

\section{Introduction}

Existing component-based methods for conducting chemical mixture health risk assessments were developed to use available experimental animal data as well as the human health effects data in the toxicological and epidemiological

\footnotetext{
* Corresponding author. Tel.: +1 804827 2058; fax: +1 8048288900 .

E-mail address: gennings@hsc.vcu.edu (C. Gennings).
}

literature (Teuschler et al., 2002). These methods generally rely on default assumptions (e.g., dose addition or response addition) which are considered reasonable but lack experimental validation (ATSDR, 2000a,b,c; U.S. EPA, 2000). Interest in understanding the potential human health effects of exposure to mixtures has increased due to recent congressional acts. The Food Quality Protection Act of 1996 directs that assessments of pesticide safety include consideration of the risk(s) associated with the cumulative effects of chemicals 
that have a common mode of action while the Safe Drinking Water Act Amendments, also of 1996, requests the development of new approaches for studying complex mixtures.

The toxicity of a mixture depends on the toxicity of the components and how the components interact with each other in a dose-dependent way. Usage and application rates of fungicides, insecticides and herbicides depend on many factors. The selection of particular pesticides and/or their application limits could be based on knowledge of which chemicals combine synergistically. In general, for industrial/regulatory decision-makers to select/change these processes, it is important to identify whether or not the components of the mixture are acting additively.

Practical methods for evaluation of the assumption of additivity in mixtures with many components have only recently been developed (e.g., Casey et al., 2004a) and are not widely available to decision makers. These statistical methods were developed to accommodate economical and practical designs for more than binary and tertiary mixtures. They are useful for low-dose exposures and reflect the toxicities associated with the relative proportions of the chemicals comprising the mixture. The experimental design used to collect the data is the 'ray design.' A ray is defined by a fixed mixing ratio of the components in a specified mixture. Environmental realism is incorporated by selection of mixing ratios that reflect the relative proportions of the chemicals in environmental mixtures. The dose-responsiveness of the mixture is evaluated in terms of the total dose/concentration of the mixture with the ratio of the components fixed. A statistical model that allows for the evaluation of the low-dose region is a threshold model (Cox, 1987). If the researcher is willing to assume the possibility of the existence of a threshold, then such a model is useful for estimating the threshold dose, below which responses are assumed to be not different from background. When used with a ray design, the analyst evaluates the dose threshold in terms of total dose for the exposure relevant mixture. Based on information/data from single components of the mixture, the predicted dose-response relationship under the assumption of additivity can be compared to that observed from the mixture. Such a strategy for evaluating mixtures has been previously described (Gennings et al., 1997, 2000, 2002; Meadows et al., 2002; Casey et al., 2004a).

The objective of this manuscript is to illustrate this strategy with a mixture of five pesticides (acephate (ACE), diazinon (DIA), chlorpyrifos (CPF), malathion (MAL) and dimethoate (DIM)). The endpoint of interest is neurotoxicity in rats as measured by a gait score dichotomized to indicate presence or absence of a gait abnormality (i.e., incoordination, loss of balance, etc.). The fixed-ratio ray under study was chosen based on the relative dietary exposure estimates of each chemical as projected by the U.S. EPA Dietary Exposure Evaluation Model (DEEM) and is given by 0.040:0.002:0.031:0.825:0.102 for ACE, DIA, CPF, MAL, and DIM, respectively. The data from the five-chemical mixture study are referred to as 'full ray' data. To evaluate the effect of one pesticide (MAL) on the dose-response relation- ship of the other four pesticides, a 'reduced ray' was evaluated experimentally in which the remaining four pesticides were fixed at the same relative proportions as in the full ray, i.e., 0.229:0.011:0.177:0:0.583 for ACE, DIA, CPF, MAL, and DIM.

\subsection{Additivity model}

The definition of additivity used herein is given by Berenbaum (1985) and is based on the classical isobolograms for the combination of two chemicals (e.g., Loewe and Muischnek, 1926; Loewe, 1953). That is, in a combination of $c$ chemicals, let $E_{i}$ represent the dose of the $i$ th component alone that yields a fixed response, and let $x_{i}$ represent the concentration/dose of the $i$ th component in combination with the $c$ agents that yields the same response. According to this definition of additivity if the substances combine additively, i.e., with zero interaction, then

$\sum_{i=1}^{c} \frac{x_{i}}{E_{i}}=1$

If the left-hand side of (1) is less than 1 , then a synergism can be claimed at the combination of interest. If the lefthand side of (1) is greater than 1, then an antagonism can be claimed at the combination. This definition of additivity is a general form for dose-addition. It should be pointed out that use of the toxic equivalency factors approach or the relative potency factors approach (Finney, 1964; U.S. EPA, 2003) assumes common slopes across the chemicals under study; the general dose-addition definition of (1) does not require such an assumption. In what follows, the additivity models are algebraically equivalent to the definition given in (1).

Response surface methodology, often supported by factorial designs, is the classical statistical experimental approach for testing for departure from additivity. Alternatively, fixedratio ray designs have been proposed (e.g., Gennings et al., 2002, Meadows et al., 2002). Fixed-ratio rays can be used to reduce the amount of experimental effort when the exposure region of interest is restricted to relevant mixing ratios. Two analysis strategies designed for use in conjunction with fixed-ratio experimental designs are illustrated here and result in similar conclusions. The first approach is similar to that described by Gennings et al. (2002) applied to a threshold additivity model, and is termed the 'single chemicals required' (SCR) method. The second approach has been described by Meadows et al. (2002) and Casey et al. (2004a) and is termed the 'single chemicals not required' (SCNR) method.

The general strategy begins by using the single chemical data to fit a threshold additivity model. The threshold additivity model is used to estimate the dose-response relationship along the fixed-ratio ray(s) of interest (in terms of total dose) under the hypothesis of additivity. This additivity model is used to provide power/sample size calculations to design the mixture study with adequate power to detect biologically meaningful interactions. The mixture data are 
then experimentally generated and fit to a threshold model in terms of total dose. For the SCR method, the mixture model is compared statistically to the predicted model under additivity. If the models are statistically different, then departure from additivity is inferred. When the studies have the power to detect a biologically meaningful departure from additivity and there is not significant evidence of departure from additivity, additivity can be claimed.

The SCNR method assumes a general parameterization of the underlying multidimensional response surface (in this case six dimensions). The result is that the terms in a polynomial model along a fixed ratio ray are associated with interaction terms in the underlying model (Meadows et al., 2002; Casey et al., 2004a). For example, a significant cubic term in the mixture model is an indication of a three-way interaction among the chemicals in the mixture.

The threshold additivity model (e.g., Gennings et al., 1997) is of the form

$$
g\left(\mu_{\mathrm{A}}\right)=\left\{\begin{array}{ll}
\left\{\begin{array}{ll}
\beta_{0} & \text { if } \sum_{i=1}^{5} \beta_{i} x_{i}<\delta \\
\beta_{0}+\sum_{i=1}^{5} \beta_{i} x_{i}-\delta & \text { if } \sum_{i=1}^{5} \beta_{i} x_{i} \geq \delta
\end{array}\right\}, \\
\text { if } \beta_{\mathrm{i}} \geq 0, \forall i & \text { if } \sum_{i=1}^{5} \beta_{i} x_{i} \geq \delta \\
\beta_{0} & \text { if } \sum_{i=1}^{5} \beta_{i} x_{i}<\delta \\
\beta_{0}+\sum_{i=1}^{5} \beta_{i} x_{i}-\delta &
\end{array}\right\},
$$

where $g(\mu)$ is the specified link function (McCullagh and Nelder, 1989) of the response of interest, $x_{i}$ is the dose of the $i$ th single chemical, $\beta_{0}$ is an unknown parameter associated with the overall intercept, $\beta_{i}$ is an unknown parameter associated with the slope of the $i$ th pesticide dose-response, $\delta$ is the unknown parameter associated with the threshold for the additivity model.

Using this model, the parameter associated with the dose of the threshold for the $i$ th chemical is given by $\delta_{i}{ }^{*}=$ $\delta / \beta_{i}, i=1, \ldots, 5$.

If the model fits a threshold outside the experimental range (resulting in an over-parameterized model), then the corresponding generalized linear model is used, i.e.,

$g\left(\mu_{\mathrm{add}}\right)=\beta_{0}+\sum_{i=1}^{5} \beta_{i} x_{i}$

Let the mixing ratio of chemicals of interest be denoted as $\left(a_{1}: a_{2}: a_{3}: a_{4}: a_{5}\right)$ such that $\sum_{i=1}^{c=5} a_{i}=1$. Following Gennings et al. (2002) and using the definition of additivity given in (1) and the additivity models in either (2) or (3), the slope along the fixed-ratio ray design under additivity is given by $\sum_{i=1}^{c} a_{i} \beta_{i}$.

The biological endpoint presented herein is based on evidence of the presence or absence of a gait abnormality (Moser,
Table 1

Parameter estimates and associated $\mathrm{p}$ values from the additivity model given in (3)

\begin{tabular}{lcll}
\hline Parameter & Estimate & Standard error & $p$ value \\
\hline$\beta_{0}$ & -7.349 & 1.502 & $<0.001$ \\
$\beta_{1}$ (ACE) & 0.2616 & 0.0574 & $<0.001$ \\
$\beta_{2}$ (DIA) & 0.0497 & 0.0116 & $<0.001$ \\
$\beta_{3}$ (CPF) & 0.3023 & 0.0640 & $<0.001$ \\
$\beta_{5}$ (DIM) & 0.2515 & 0.0674 & $<0.001$ \\
\hline
\end{tabular}

The slope for MAL was not included as no gait abnormalities were observed in the experimental range for MAL. The estimate for the scale parameter was $\hat{\tau}=1.137$

1995). To appropriately constrain the probability of a response to be between 0 and 1 , a logit link function was used, i.e., $g(\mu)=\log (\mu /(1-\mu))$. It was assumed throughout that the variance changes as a binomial random variable such that $\operatorname{Var}(Y)=\tau \mu(1-\mu)$ (e.g., McCullagh and Nelder, 1989).

Parameter estimates for the threshold model in (2) are found using the maximum quasi-likelihood criterion (e.g., McCullagh and Nelder, 1989) in a Nelder Mead algorithm (Nelder and Mead, 1965). Parameter estimates for the generalized linear model given in (3) were found using the maximum quasi-likelihood criterion using a Fisher scoring algorithm in Proc GENMOD in SAS (version 8.2). A moment estimate for $\tau$ (McCullagh and Nelder, 1989) was used. Adequacy of the fit of the model to the data was assessed graphically and by comparing the scaled deviance to a $\chi_{\mathrm{N}-\mathrm{p}}^{2}$ distribution where $N$ is the total sample size and $p$ is the number of model parameters.

Raw data used for these analyses are provided in the Appendix. Dose-response curves for the five individual pesticides were generated and the threshold model given in (2) was fit to these data. The dose threshold estimates were all outside the experimental region; thus the corresponding generalized linear model given in (3) was used as the additivity model. Parameter estimates and associated $p$ values are provided in Table 1. Plots of the observed and model predicted responses are provided in Fig. 1. The single chemical data are adequately represented by the additivity model ( $p=0.937$, using scaled deviance as an assessment of fit).

In addition to gait score, other biological and neurochemical endpoints were taken. Sample size estimates ( $n=12 /$ mixture group) were calculated for motor activity data to detect a $25 \%$ decrease (i.e., more negative) in the slope of the dose-response curve along the fixed-ratio ray from that under additivity with $70 \%$ power using a Wald-type test (as described in Casey et al., 2004b) with a two-sided test with $5 \%$ significance. When the decrease in the slope was $30 \%$, the resulting power was $84 \%$.

As the mixture studies were not run concurrently with the single chemical studies, it was important to demonstrate that the single chemical dose-response curves do not shift across the studies (mixture and single chemical). 'Positive control' values of the single chemicals (two to three doses of each individual chemical except malathion, one dose) were included in the mixture studies for this purpose. See Appendix for the 

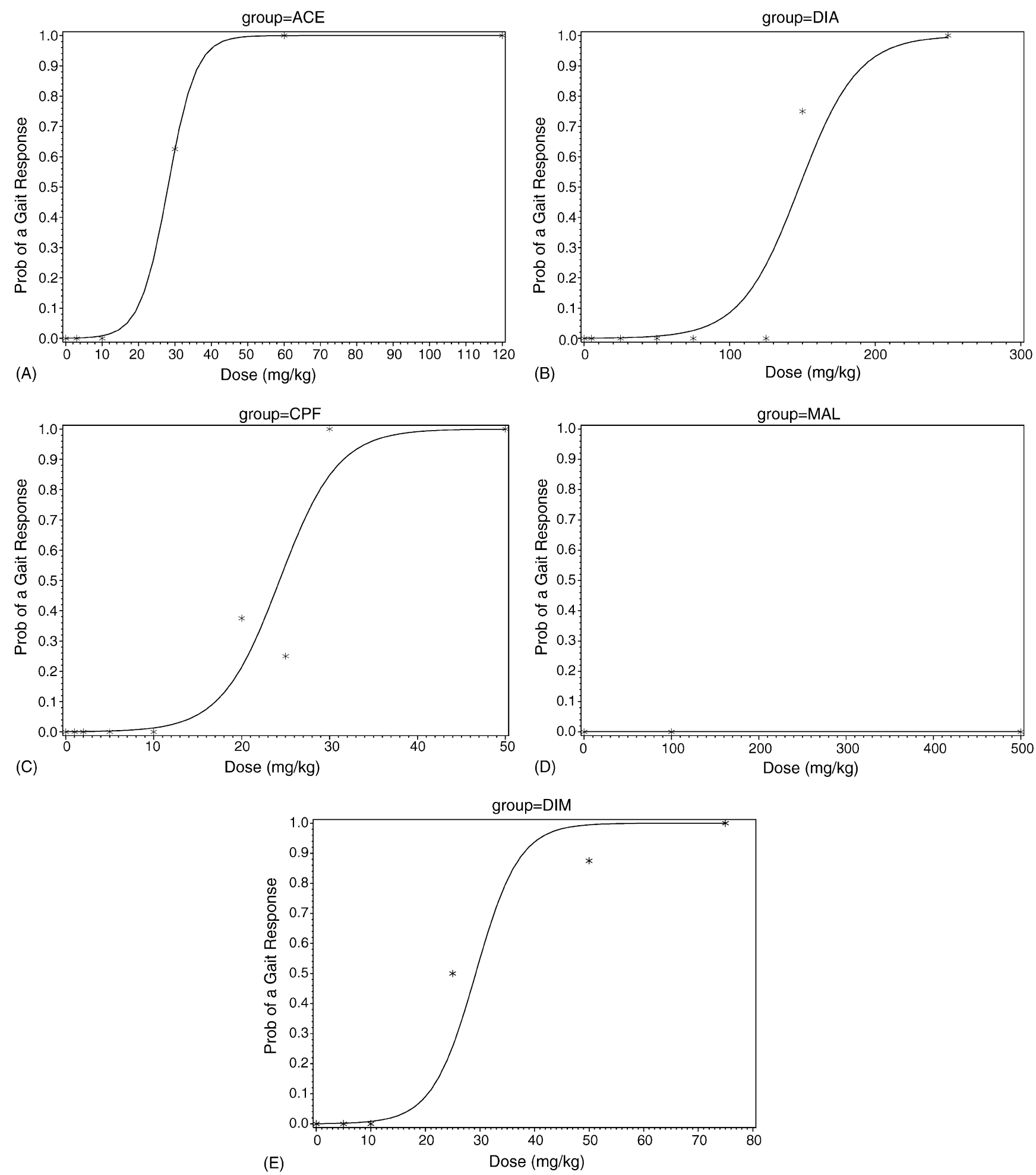

Fig. 1. Observed and predicted responses from the additivity model with estimates given in Table 1 for (A) ACE, (B) DIA, (C) CPF, (D) MAL, and (E) DIM.

raw data. The mixture experiment consisted of a vehicle control $(n=14)$ and six total dose mixture concentrations $(10,55$, $100,200,300$ and $450 \mathrm{mg} / \mathrm{kg} ; n=12$ rats per dose group) at a fixed mixing ratio ray of 0.040:0.002:0.031:0.825:0.102 for ACE, DIA, CPF, MAL, and DIM. Similarly, the design for a second fixed-ratio ray was based on a reduced number of the pesticides (omitting MAL) with the remaining pesticides at the same actual doses and relative proportions as in the 'full' mixture study. The experiment consisted of a vehicle control $(n=8)$, positive controls ( $n=8$ per group) for each in- 
dividual chemical, and six total dose mixture concentrations $(1.75,9.6,17.5,35,52.5$, and $78.8 \mathrm{mg} / \mathrm{kg} ; n=12$ rats per dose group) at a fixed mixing ratio ray of 0.229:0.011:0.177:0.583 for ACE, DIA, CPF, and DIM. The doses used in this study were chosen such that at least two doses in each of the individual dose-response curves were ineffective, so that the lower end of the dose range could be characterized. Furthermore, even the highest doses of individual chemicals in the mixture were at or below LOELs defined in the individual dose-response curves, the only exception being dimethoate (for which the highest dose in the mixture produced considerable effects when given alone). All doses were administered by oral gavage at $2 \mathrm{ml} / \mathrm{kg}$. Because of different solubilities, each rat received a dose of acephate dissolved in water immediately followed by a dose of the remaining pesticides in a 5\% ethanol:95\% corn oil vehicle.

\section{2. 'Single chemicals required' method}

Following the logic of Gennings et al. (2002), for comparison to the additivity model along the fixed-ratio rays given by (3), the mixture data were fit to a 'mixture model' along each ray in terms of total dose.

$g\left(\mu_{\text {mix }}\right)=\beta_{0}+\theta_{1}^{*} t$

In order to achieve adequate fit to the data, higher order terms in total dose are added to (4) when necessary.

The five vehicle control groups for the single chemical data and the vehicle control groups for the two mixture studies resulted in no gait abnormalities. Therefore, since the background rate is similar across the studies, the additivity model in (3) and the mixture model in (4) for both the full and reduced rays were fit simultaneously in an overall model with a common background parameter, $\beta_{0}$. In addition, the two mixture studies included at least one 'positive control' group for each of the single chemicals alone. Preliminary analyses (not shown) of all of the data combined indicated a discrepancy in response at the $30 \mathrm{mg} / \mathrm{kg}$ dose group of CPF. In the single chemical study, all eight animals demonstrated a gait abnormality at this dose group; for the full ray mixture study, none of the six animals responded at this dose group of CPF alone. With the exception of this one positive control dose group, the remaining data (including two other CPF positive control values of 10 and $20 \mathrm{mg} / \mathrm{kg}$ ) did not demonstrate evidence of a shift in the dose-response curves from the original single chemical studies using the positive control data $(p=0.235)$. In order to proceed to demonstrate the methods, the overall model was based on the single chemical data and the mixture data combined. It should be noted that even with a slight shift in the CPF curve, only $3.1 \%$ of the mixture was CPF.

The overall model included a common intercept term, linear terms for each of the single chemicals (excluding MAL), a linear and quadratic term in total dose for the full ray, and a linear term in total dose for the reduced ray (described above),
Table 2

Parameter estimates and associated $p$ values from the full SCR analysis

\begin{tabular}{lclr}
\hline Parameter & Estimate & Standard error & $p$ value \\
\hline$\beta_{0}$ & -4.254 & 0.5831 & $<0.001$ \\
$\beta_{1}$ ACE & 0.1571 & 0.0392 & $<0.001$ \\
$\beta_{2}$ DIA & 0.0276 & 0.0060 & $<0.001$ \\
$\beta_{3}$ CPF & 0.1782 & 0.0334 & $<0.001$ \\
$\beta_{5}$ DIM & 0.1425 & 0.0363 & $<0.001$ \\
$\theta_{1(1)}$ full ray linear & 0.0506 & 0.0110 & $<0.001$ \\
$\theta_{2(1)}$ full ray quadratic & -0.000076 & 0.000027 & 0.005 \\
$\theta_{1(2)}$ reduced ray linear & 0.1242 & 0.0232 & $<0.001$ \\
\hline
\end{tabular}

The estimate for the scale parameter was $\hat{\tau}=1.48$. The overall hypothesis of additivity was rejected ( $p=0.036$, with 3 degrees of freedom (d.f.)). The hypothesis of additivity along the full ray was rejected ( $p=0.053$, with 2 d.f.) but was not rejected along the reduced ray $(p=0.314,1$ d.f.). The hypothesis of no malathion effect was rejected ( $p=0.014,2$ d.f.).

which adequately represented the data $(p=0.900$, i.e., no indication of lack of fit). The resulting parameter estimates and associated $p$ values are provided in Table 2. As the linear parameters associated with total dose along both the full and reduced rays are significant, there is evidence that as the total dose of the mixture increases, the probability of a gait abnormality also increases.

From the parameter estimates given in Table 2, plots of observed and predicted responses along both fixed-ratio mixture rays are provided (Fig. 2) based on the mixture model and under the assumption of additivity. Following the approach in Gennings et al. (2002), as the curve predicted using the mixture model for the full mixture ray falls significantly above the predicted dose-response curve under the assumption of additivity (Fig. 2A) ( $p=0.053)$, it can be inferred that the overall effect of the specified fixed-ratio of the five pesticides is associated with a greater than additive, or synergistic, relationship. Further, there is not a significant difference (Fig. 2B) in the dose-response curves from the mixture model and that predicted under the assumption of additivity for the mixture (omitting MAL) along the reduced ray $(p=0.314)$. Following the logic of Casey et al. (2004a), this indicates that the interaction (i.e., synergy) in the full ray may be associated with the presence of MAL. Casey et al. (2004a) developed a method for combining the results from multiple rays with the same relative ratios (like the full and reduced rays here) in the same figure. If the adjusted dose-response curve from the full ray is identical to the dose-response curve on the reduced ray, then it indicates the chemical or subset of chemicals that were removed in the reduced ray do not interact with the remaining chemicals. For these data, testing for the difference in the corrected and corresponding parameters from the full and reduced rays (Casey et al., 2004a) indicates that the presence of MAL significantly $(p=0.014)$ changes the shape of the dose-response curve in the full ray. This is elucidated in Fig. 2C where the dashed curve corresponds to the adjusted dose-response curve from the five-pesticide mixture plotted with the dose-response curve without MAL (solid curve). 

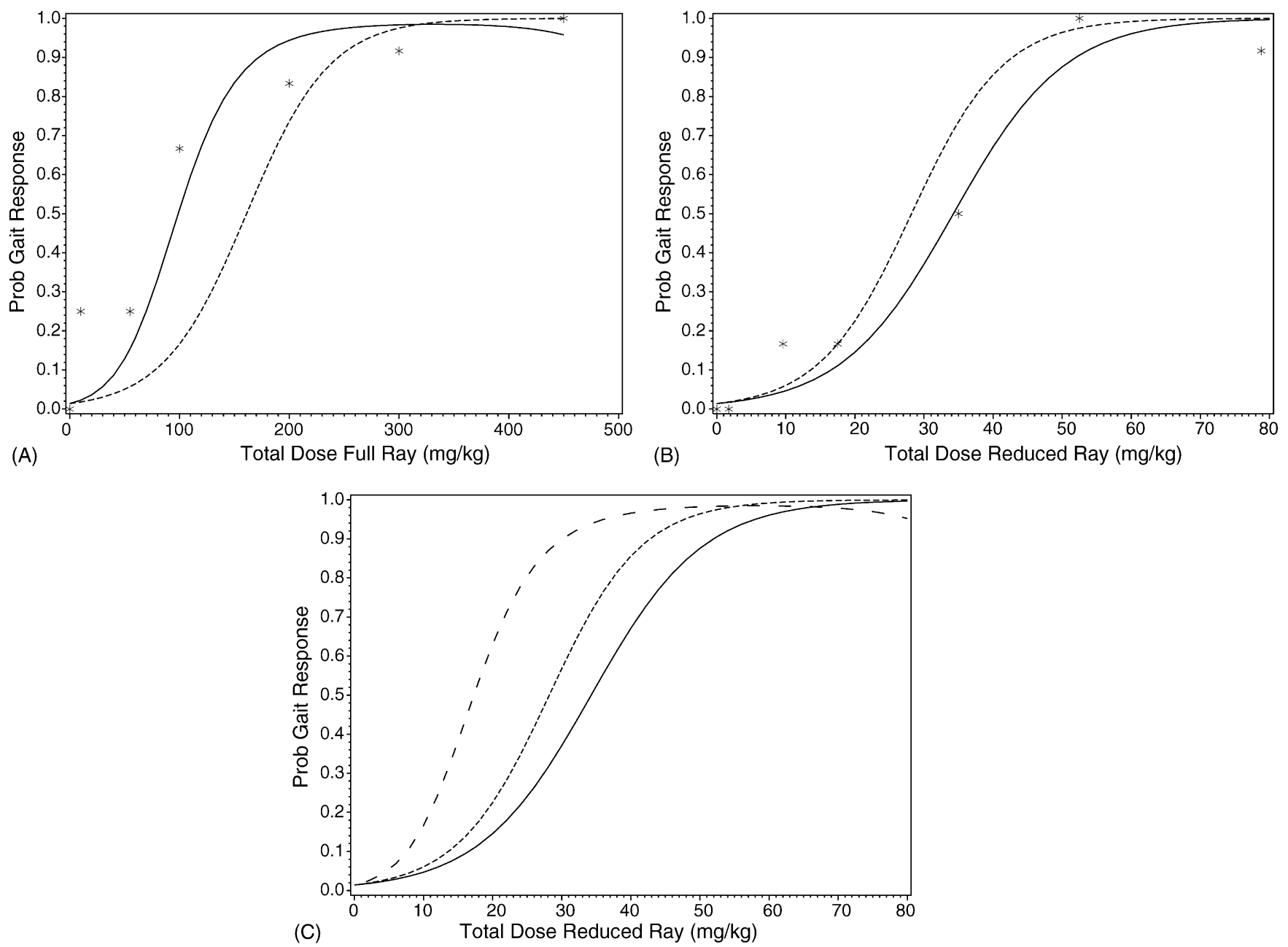

Fig. 2. Observed $\left(^{*}\right)$ and predicted responses based on the SCR mixture model (solid line) and assuming additivity (dotted line) as a function of total dose $(\mathrm{mg} / \mathrm{kg})$ for the (A) full ray with all five chemicals and (B) for the reduced ray with MAL omitted. Part C includes the predicted response under additivity (dotted line) and based on the reduced ray mixture model (solid line) without MAL as given in B. In addition, part C includes an adjusted curve (dashed line) from the full ray mixture data (including MAL).

These dose-response curves are significantly different due to the presence of MAL. The total dose that is associated with a $50 \%$ response $\left(\mathrm{ED}_{50}\right)$ for the four pesticides in the absence of malathion is about $34 \mathrm{mg} / \mathrm{kg}$. In the presence of malathion, the total dose of the other four pesticides associated with a $50 \%$ response is roughly $17 \mathrm{mg} / \mathrm{kg}$. This two-fold difference indicates the magnitude of the effect of the synergism at a $50 \%$ response level. It is apparent from Fig. $2 \mathrm{C}$ that the magnitude of the synergism is dependent on the level of the effect (i.e., $10,50 \%$, etc.).

\section{3. 'Single chemicals not required' method}

Following the methods of Meadows et al. (2002) and Casey et al. (2004a), a comparison analysis strategy to the SCR method is based on an assumption of a general parameterization of the underlying six-dimensional (five chemicals and one response dimension) response surface. Meadows et al. (2002) showed that polynomial terms of degree two or greater for the model along a fixed-ratio ray are associated with interactions among the chemicals in a mixture. Casey et al. (2004a) generalized this result to the case of multiple mixture rays and termed this approach the 'single chemicals not required' (SCNR) method. As the name suggests, the method is applicable to the case where single agent data are not available for estimation of the additivity relationship. This information is 'replaced' with the assumption of the parametric form of the underlying response surface. However, if single agent data are available, they can be used to support the estimation of the corresponding parameters in the model.

In comparison to the mixture model in (3), here we allow for higher degree terms, which are interpreted as being associated with interactions. The interaction model for a mixture of $c$ chemicals is given by

$g\left(\mu_{\text {mix }}\right)=\beta_{0}+\sum_{i=1}^{c} \beta_{i} t^{i}$ 

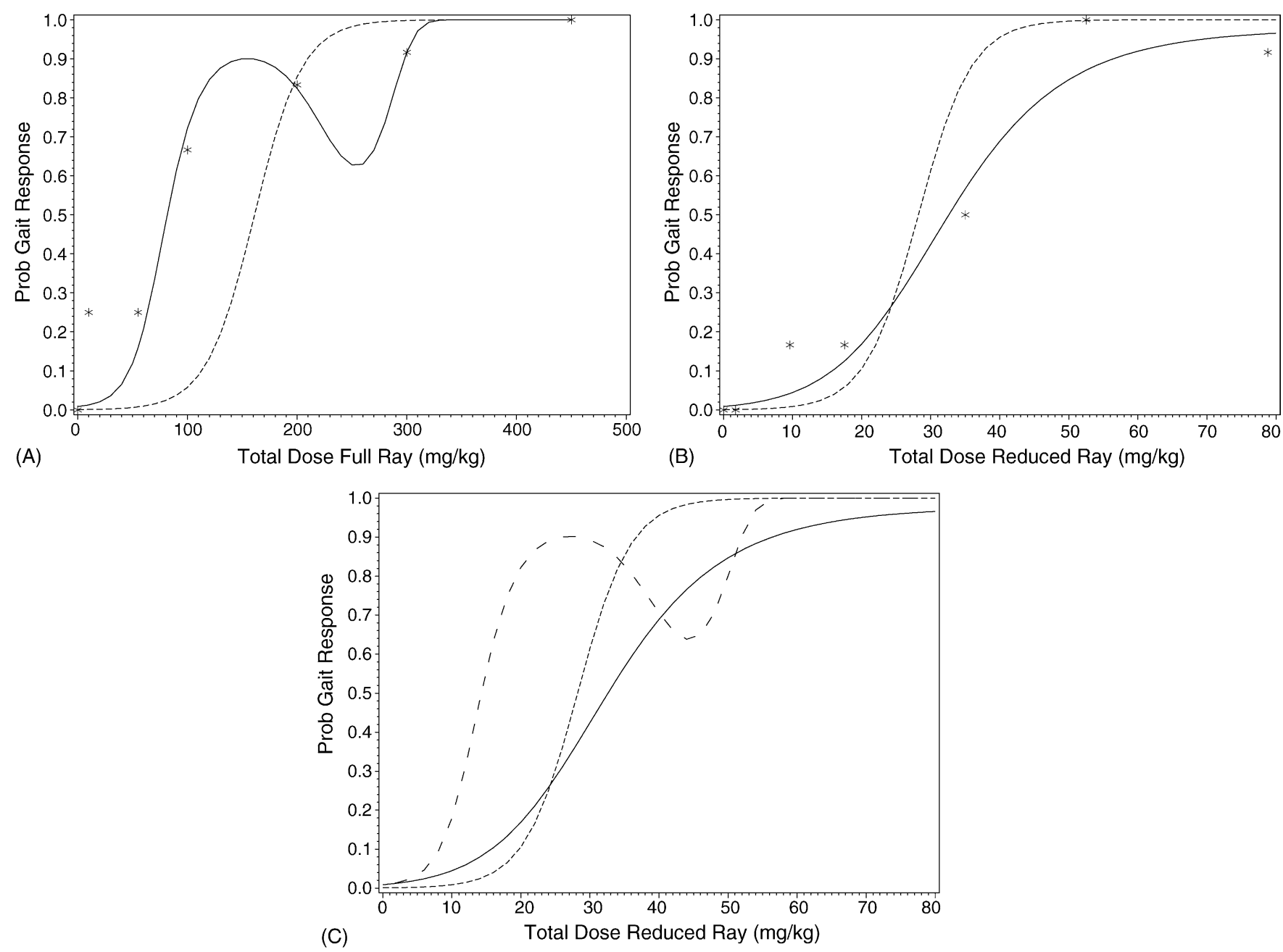

Fig. 3. Observed $\left({ }^{*}\right)$ and predicted responses based on the SCNR mixture model (solid line) and assuming additivity (dotted line) as a function of total dose $(\mathrm{mg} / \mathrm{kg})$ for the (A) full ray with all five chemicals and (B) for the reduced ray with MAL omitted. Part C includes the predicted response under additivity (dotted line) and based on the reduced ray mixture model (solid line) without MAL as given in B. In addition, part C includes an adjusted curve (dashed line) from the full ray mixture data (including MAL).

For example, for data from the full ray with five pesticides, a polynomial model with up to a fifth degree term was considered; for the data from the reduced ray with four pesticides in the mixture, a polynomial model with up to a fourth degree term was considered. The significance of the $i$ th-degree term is interpreted as evidence of an $i$ th-way interaction $(i=2, \ldots, \mathrm{c})$, but is not suggestive of which $i$ components are interacting. For example, in the mixture analysis of five pesticides, (for ease of notation denoted as A, B, C, D, E) there are five possible four-way interactions: $\mathrm{ABCD}, \mathrm{ABCE}$, $\mathrm{ABDE}, \mathrm{ACDE}$, and BCDE. The significance of the fourth degree term in the model given in (5) indicates that one or more of these four-way interactions are present. Further experimentation is needed in order to infer which components are interacting (Meadows et al., 2002; Casey et al., 2004a). Estimation of model parameters follows similarly to that described in the previous section.

The initial model fit to the gait score data had a common intercept term across the studies (five single chemical and two mixture studies), linear terms for each single chemical study for estimating the additivity response, a fifth-degree polynomial for the full ray data, and a fourth-degree polynomial for the reduced ray data. All of the data are used to estimate the additive part of the model, but only the mixture data were used to estimate the corresponding higher-degree terms. From this model, the simultaneous test for the significance of the higher-degree terms is equivalent to a test of additivity. For these binary gait score data, there was an indication of departure from additivity ( $p=0.058$, with 7 d.f.). In order to make the model more parsimonious, we used a backward elimination approach to delete terms that were not statistically significant. We reduced the model for the full ray first while keeping the reduced ray fully parameterized. Once the model for the full ray was reduced to having only significant terms, then the model for the reduced ray was simplified. None of the higher-order terms for the reduced ray were significant; however, we kept the pure quadratic term for flexibility in the model. 
Table 3

Parameter estimates and associated $p$ values from the final SCNR analysis

\begin{tabular}{lclr}
\hline Parameter & Estimate & Standard error & $p$ value \\
\hline$\beta_{0}$ & -4.723 & 0.726 & $<0.001$ \\
$\beta_{1}$ ACE & 0.1778 & 0.0440 & $<0.001$ \\
$\beta_{2}$ DIA & 0.0309 & 0.00697 & $<0.001$ \\
$\beta_{3}$ CPF & 0.1989 & 0.0391 & $<0.001$ \\
$\beta_{5}$ DIM & 0.1699 & 0.0443 & $<0.001$ \\
$\theta_{2(1)}$ full ray quadratic & 0.000751 & 0.000308 & 0.015 \\
$\theta_{3(1)}$ full ray cubic & -0.00000605 & 0.00000269 & 0.025 \\
$\theta_{4(1)}$ full ray quartic & 0.0000000116 & 0.00000000566 & 0.041 \\
$\theta_{2(2)}$ reduced ray quadratic & -0.000930 & 0.000512 & 0.070
\end{tabular}

The estimate for the scale parameter was $\hat{\tau}=1.51$. The overall hypothesis of additivity was rejected ( $p=0.021$, with 4 d.f.). The hypothesis of additivity along the full ray was rejected ( $p=0.046$, with 3 d.f.) but was not rejected along the reduced ray $(p=0.090,1$ d.f.). The hypothesis of no malathion effect was rejected $(p=0.019,3$ d.f.).

The resulting parameter estimates for the 'final' model, standard errors, and $p$ values are provided in Table 3. As all four of the linear terms are positive and significant, we conclude that as any of the doses of the chemicals (except for MAL) increase, the probability of a gait abnormality also increases. The overall test of additivity was rejected indicating the significance of at least one higher degree term $(p=0.021$, 4 d.f.). Fig. 3A presents a plot of the dose-response curve in terms of total dose for the predicted model compared to what it would be under additivity. Starting with the highest degree term, since the fourth degree term is significant for the full ray, we conclude that at least one four-way interaction exists. Without further information, it is not evident which four chemicals are involved.

By comparison, none of the higher degree terms are significant along the reduced ray $(p=0.090)$. Therefore, there was no evidence of departure from additivity among the four pesticides (ACE, DIA, CPF, and DIM; see Fig. 3B). Recall the relative ratios of these pesticides are equivalent to those used in the full ray. Since there is an indication of at least one fourway interaction on the full ray where MAL is present, and no indication of departure from additivity on the reduced ray where MAL is absent, there is evidence to suggest that MAL interacts with at least some or all of the other four pesticides. Following the work of Casey et al. (2004a), the hypothesis of no MAL interaction with the other pesticides was rejected ( $p=0.019,3$ d.f.). Fig. 3C depicts the same two curves as in Fig. 3B with the additional curve (dotted) obtained from the full ray, corrected for the proportion of the pesticides that were included in the reduced ray (here $1-0.825$ since MAL was $82.5 \%$ of the mixture). The difference in the dotted and solid curves indicates the effect MAL has on the mixture.

\section{Discussion}

In summary, both analysis strategies (SCR and SCNR) resulted in similar conclusions, namely that (1) ACE, DIA,
CPF and DIM when given alone had a significant effect on a gait abnormality and MAL was not dose-responsive, (2) there is a significant interaction among the five pesticides along the fixed-ratio mixture ray which is associated with a synergistic effect, (3) there is not a significant departure from additivity among the four pesticides (omitting MAL) along the reduced mixture ray, and (4) adjusted comparisons across the full and reduced rays indicate that MAL interacts with the other pesticides. This endpoint is part of a larger study which included motor activity and cholinesterase inhibition. Those analyses are being completed and will be presented in a future manuscript; however, preliminary analyses support a conclusion of greater-than-additive responses.

More generally, the methods described here can be readily generalized to other types of chemicals, different numbers of components in a mixture, and to different types of endpoints. The methods based on exposure relevant fixed ratio ray designs allow for dramatic decreases in the required experimental effort for studying mixtures of many chemicals as compared to the classical factorial designs. The design for the study of the mixture of five pesticides essentially required adequate support for seven dose-response curves (five single chemicals alone and two total dose mixture rays). Positive controls were added in order to demonstrate the comparability of the studies not run concurrently. By comparison a full factorial design for five chemicals with reasonable support of the shape of the dose-response curve (i.e., not just two dose points per chemical) would have required more experimental effort. For example, a full factorial design for a mixture of five chemicals each at four levels (including control) has 1024 dose groups, Fractionated factorial designs are available (e.g., Groten et al., 1997). But, as the number of chemicals in the mixture increases, the experimental effort for even these designs quickly becomes impractical.

The single chemical and mixture data were combined for the analyses presented here. There was some concern about the discrepancy between the observed responses from $\mathrm{CPF}$ at $30 \mathrm{mg} / \mathrm{kg}$ in the original single chemical study ( 8 out of 8 responded) and as a 'positive control' in the full ray mixture study ( 0 out of 6 responded; see appendix). All of the other positive control values were in agreement with the original single chemical studies $(p=0.235)$. The positive control values from the full mixture ray study for CPF may indicate a shift to the right for the active part of the CPF dose-response curve. However, analysis of the mixture data (see Fig. 2A) indicated a shift to the left from that predicted from the original single chemical data under the hypothesis of additivity. That is, the conclusion of a greater than additive effect was not due to a shift in the CPF effect in the mixture as it was in the opposite direction.

Meadows et al. (2004) describe an example where the single chemical data were not appropriate for comparison to the mixture data. In such cases, the SCNR method may be used to estimate departure from additivity with only mixture data 
along a ray to support the estimation of the model parameters. In screening methods for mixtures of many chemicals, it may not be feasible to supply dose-response curves for each component in the mixture. By making the assumption of a parameterization for the underlying response surface, analysis of data along a fixed-ratio ray may result in hypothesis testing for departure from additivity.

An important feature to the analysis strategies is the power and sample size calculations for the mixture study. The single chemical data were initially evaluated using an additivity model. Power and sample size methods (Meadows et al., 2002; Casey et al., 2004b) were used to determine dose locations and sample sizes to yield an acceptable level of power to detect departure from additivity to a biologically important degree. Here a change in the parameter associated with the slope of at least $25 \%$ from that predicted under additivity was considered biologically meaningful. If the study is adequately powered and no evidence of departure from additivity is found, then additivity can be claimed. By comparison, if the study is not adequately powered, then lack of evidence of departure from additivity may be a power problem and not an indication of additivity.

Using both the SCR and SCNR methods departures from additivity were found for the given mixture of the five pesticides. This claim of interaction applies to the specified mixing ratio and may not be true for other mixing ratios. The characterization of the interaction may also change along a fixedratio ray. For example, Gennings et al. (2002) demonstrated in a mixture of four metals that the relationship among the chemicals changed from synergistic, to additive, to antagonistic along the specified ray. Casey et al. (2004a) developed methods of determining the location of interaction for a fixed ratio of chemicals through use of simultaneous confidence regions.

The analysis of gait score described here, Figs. 2C and 3C suggest a malathion effect at low total doses. An interaction with malathion was not totally unexpected, since a frequently-cited example of OP mixtures is the potentiation of malathion toxicity by other OPs, which inhibit the carboxylesterase-mediated hydrolysis of malathion (e.g., Murphy and DuBois, 1957). Given the large amount of carboxylesterases in the body, such a kinetic interaction would be expected more at higher dose levels than were observed in the present study.

With support from government agencies, industry, and scientific societies (including the National Institute of Environmental Health Sciences, the American Chemistry Council, the U.S. Environmental Protection Agency, the Society for Environmental Toxicology and Chemistry, and the Chlorine Chemistry Council) the Society of Toxicology recently spearheaded a series of activities intended to advance the scientific understanding of environmental mixtures. An expert Working Group was charged with evaluating the state of the science on environmental mixtures, providing a conceptual framework for future mixtures research, and suggesting potential areas for empirical and mechanistic experimentation. A resulting white paper was published (Teuschler et al.,
2002) which outlined three key ideas with extended discussion. The first key idea stated that "toxicology experiments on whole mixtures or mixtures components should include doses at or below the no-observed-effect levels [NOAELs/NOELs] for individual mixture components. The mixture components that are tested and their relative proportions in the mixture also should reflect those seen in environmental samples. In addition, the impact of the unidentified materials in the mixtures should be considered." The SCR and SCNR methods accommodate this directive as they are based on a ray design such that the mixing ratios of the chemicals in the mixture may be determined by relevant/environmental ratios. Threshold models may be used to describe the dose-response relationship at or below the no-observed-effect levels. In addition, these methods facilitate study of the unidentified materials in a mixture through comparisons of 'full' and 'reduced' rays where the unidentified materials are included and excluded, respectively. The resulting analysis is analogous to that illustrated here with and without MAL in the mixture.

Finally, the risk assessment process is complicated by the fact that environmental exposures frequently may involve mixtures of chemicals rather than a single compound. Public concern about such mixtures led to new requirements under the Food Quality Protection Act (1996) to assess risk of pesticide mixtures that have a common mode of toxicity. The methods described here may be useful in providing an experimentally feasible way of studying exposure-relevant mixtures instead of regulating chemicals based on default assumptions of additivity. In addition, if departure from additivity is concluded, the impact of important components of the mixture can be assessed by comparison of 'full' and judiciously chosen 'reduced' rays of interest.

\section{Acknowledgments}

This research was supported by cooperative agreements from the U.S. EPA (NCEA-Cincinnati, \#CR-827208; NHEERL-RTP, \#CR-82811401-0). We would like to thank Mike DeVito, Linda Birnbaum, David Herr, and Linda Teuschler for their insightful reviews of the manuscript. Disclaimer: This manuscript has been reviewed by the National Health and Environmental Effects Research Laboratory, U.S. Environmental Protection Agency, and approved for publication. Approval does not signify that the contents necessarily reflect the views of the Agency nor does mention of trade names or commercial products constitute endorsement or recommendation for use.

\section{Appendix A}

Summary statistics from single chemical and mixture experiments: Table A1 
Table A1

The number of responders $(r)$ represent the number of rats with gait scores $>1$ within each dose group

\begin{tabular}{|c|c|c|c|}
\hline Group & Dose (mg/kg) & $r$ & $N$ \\
\hline \multicolumn{4}{|c|}{ Single chemical data } \\
\hline ACE & 0 & 0 & 8 \\
\hline ACE & 3 & 0 & 8 \\
\hline $\mathrm{ACE}$ & 10 & 0 & 8 \\
\hline $\mathrm{ACE}$ & 30 & 5 & 8 \\
\hline ACE & 60 & 8 & 8 \\
\hline ACE & 120 & 8 & 8 \\
\hline $\mathrm{CPF}$ & 0 & 0 & 16 \\
\hline $\mathrm{CPF}$ & 1 & 0 & 8 \\
\hline $\mathrm{CPF}$ & 2 & 0 & 8 \\
\hline $\mathrm{CPF}$ & 5 & 0 & 8 \\
\hline $\mathrm{CPF}$ & 10 & 0 & 16 \\
\hline $\mathrm{CPF}$ & 20 & 3 & 8 \\
\hline $\mathrm{CPF}$ & 25 & 2 & 8 \\
\hline $\mathrm{CPF}$ & 30 & 8 & 8 \\
\hline $\mathrm{CPF}$ & 50 & 16 & 16 \\
\hline DIA & 0 & 0 & 16 \\
\hline DIA & 5 & 0 & 16 \\
\hline DIA & 25 & 0 & 16 \\
\hline DIA & 50 & 0 & 8 \\
\hline DIA & 75 & 0 & 16 \\
\hline DIA & 125 & 0 & 8 \\
\hline DIA & 150 & 6 & 8 \\
\hline DIA & 250 & 8 & 8 \\
\hline DIM & 0 & 0 & 8 \\
\hline DIM & 5 & 0 & 8 \\
\hline DIM & 10 & 0 & 8 \\
\hline DIM & 25 & 4 & 8 \\
\hline DIM & 50 & 7 & 8 \\
\hline DIM & 75 & 8 & 8 \\
\hline MAL & 0 & 0 & 8 \\
\hline MAL & 100 & 0 & 8 \\
\hline MAL & 500 & 0 & 8 \\
\hline Group & Total dose $(\mathrm{mg} / \mathrm{kg})$ & $r$ & $N$ \\
\hline
\end{tabular}

Mixture data

First mixture ray ('full' ray) ${ }^{\mathrm{a}}$

Positive control data

$\begin{array}{lrrr}\text { ACE } & 6 & 0 & 6 \\ \text { ACE } & 30 & 3 & 14 \\ \text { CPF } & 10 & 0 & 6 \\ \text { CPF } & 20 & 1 & 8 \\ \text { CPF } & 30 & 0 & 6 \\ \text { DIA } & 50 & 0 & 6 \\ \text { DIA } & 125 & 1 & 14 \\ \text { DIM } & 10 & 0 & 6 \\ \text { DIM } & 25 & 1 & 8 \\ \text { DIM } & 50 & 6 & 6 \\ \text { MAL } & 350 & 0 & 14 \\ \text { Mixture data } & & & \\ \text { CON } & 0 & 0 & 14 \\ \text { MIX } & 10 & 3 & 12 \\ \text { MIX } & 55 & 3 & 12 \\ \text { MIX } & 100 & 8 & 12 \\ \text { MIX } & 200 & 10 & 12 \\ \text { MIX } & 300 & 11 & 12 \\ \text { MIX } & 450 & 12 & 12\end{array}$

Table A1 (Continued)

\begin{tabular}{lccc}
\hline Group & Total dose $(\mathrm{mg} / \mathrm{kg})$ & $r$ & $N$ \\
\hline \multicolumn{2}{c}{ Second mixture ray ('reduced' ray) } & & \\
Positive control data & 30 & 3 & 8 \\
ACE & 20 & 0 & 8 \\
CPF & 125 & 0 & 8 \\
DIA & 25 & 3 & 8 \\
DIM & & & \\
Mixture data & 0 & 0 & 8 \\
CON & 1.75 & 0 & 12 \\
MIX & 9.6 & 2 & 12 \\
MIX & 17.5 & 2 & 12 \\
MIX & 35 & 6 & 12 \\
MIX & 52.5 & 12 & 12 \\
MIX & 78.8 & 11 & 12 \\
MIX &
\end{tabular}

${ }^{a}$ The total doses for the mixture studies are based on a fixed-ratio of the five pesticides. For the 'full' ray where all five pesticides are included the mixing ratio is $(0.040: 0.002: 0.031: 0.825: 0.102)$ for (ACE: DIA:CPF:MAL:DIM), respectively.

${ }^{b}$ For the 'reduced' ray the mixing ratio is (0.229:0.011:0.177: 0.000:0.583) for (ACE:DIA:CPF:MAL:DIM), respectively.

\section{References}

ATSDR, 2000a. Agency for Toxic Substances and Disease Registry. Guidance Manual for the Assessment of Joint Toxic Action of Chemical Mixtures.

ATSDR, 2000b. Agency for Toxic Substances and Disease Registry. Interaction Profile for: Chlorinated Dibenzo-p-dioxins, Hexachlorobenzene, $p, p^{\prime}$-DDE, and Methylmercury.

ATSDR, 2000c. Agency for Toxic Substances and Disease Registry. Guidance for the Preparation of an Interaction Profile.

Berenbaum, M.C., 1985. The expected effect of a combination of agents: the general solution. J. Theor. Biol. 114, 413-431.

Casey, M., Gennings, C., Carter Jr., W.H., Moser, V.C., Simmons, J.E., 2004a. Detecting interaction(s) and assessing the impact of component subsets in a chemical mixture using fixed-ratio mixture ray designs. J. Agric. Biol. Environ. Stat, in press.

Casey, M., Gennings, C., Carter Jr., W.H., Moser, V.C., Simmons, J.E., 2004b. Power and sample size calculations for linear hypotheses associated with mixtures of many components using fixed-ratio ray designs. J. Environ. Ecol. Stat., under review.

Cox, C., 1987. Threshold dose-response models in toxicology. Biometrics 43, 511-523.

Finney, D.J., 1964. Probit Analysis: A Statistical Treatment of the Sigmoid Response Curve, second ed. University Press, Cambridge.

Gennings, C., Schwartz, P., Carter Jr., W.H., Simmons, J.E., 1997. Detection of departures from additivity in mixtures of many chemicals with a threshold model. J. Agric. Biol. Environ. Stat. 2, 198-211.

Gennings, C., Schwartz, P., Carter Jr., W.H., Simmons, J.E., 2000. Erratum: Detection of departures from additivity in mixtures of many chemical with a threshold model. J. Agric. Biol. Environ. Stat. 5, 257-259.

Gennings, C., Carter Jr., W.H., Campain, J.A., Bae, D., Yang, R.S.H., 2002. Statistical analysis of interactive cytotoxicity in human epidermal heratinocytes following exposure to a mixture of four metals. J. Agric. Biol. Environ. Stat. 7, 58-73.

Groten, J.P., Schoen, E.D., van Balderen, P.J., Kuper, C.F., van Zorge, J.A., Feron, V.J., 1997. Subacute toxicity of a mixture of nine chemicals in rats: detecting interactive effects with a fractionated two-level factorial design. Fundam. Appl. Toxicol. 36, 15-29. 
Loewe, S., Muischnek, H., 1926. Uber kombinationswirkunger. I. Mitteilung: Hiltsmittel der gragstellung. Naunyn-Schmiedebergs,. Arch. Pharmacol. 114, 313-326.

Loewe, S., 1953. The problem of synergism and antagonism of combined drugs. Arzneimittle Forshung. 3, 285-290.

McCullagh, P., Nelder, J.A., 1989. Generalized Linear Models, second ed. Chapman and Hall, New York.

Meadows, S.L., Gennings, C., Carter Jr., W.H., Bae, D.-S., 2002. Experimental designs for mixtures of chemicals along fixed ratio rays. Environ. Health Perspect. 110 (6), 979-983.

Meadows, S.L., Gennings, C., Carter Jr., W.H., 2004. Analysis of drugs/chemicals along a fixed-ratio ray without single chemical data to support an additivity model. J. Agric. Biol. Environ. Stat., in press.

Moser, V.C., 1995. Comparisons of the acute effects of cholinesterase inhibitors using a neurobehavioral screening battery in rats. Neurotoxicol. Teratol. 17, 617-625.

Murphy, S.D., DuBois, K.P., 1957. Quantitative measurement of inhibition of the enzymatic detoxification of malathion by EPN (ethyl p-nitrophenyl thionobenzene phosphate). Proc. Soc. Exp. Biol. Med. 96, 813-818.

Nelder, J.A., Mead, R., 1965. A simplex method for function minimization. Comput. J. 7, 308-313.

SAS Institute Inc, 2004. Version 8.2. Cary, NC.

Teuschler, L., Klaunig, J., Carney, E., Chambers, J., Connolly, R., Gennings, C., Giesy, J., Hertzberg, R., Klaassen, C., Kodell, R., Paustenbach, D., Yang, R., 2002. Support of science-based decisions concerning the evaluation of the toxicology of mixtures: a new beginning. Regul. Toxicol. 36, 34-39.

U.S. EPA, 2000. United States Environmental Protection Agency. Supplementary Guidance for Conducting Health Risk Assessment of Chemical Mixtures. EPA/630/R-00/002.

U.S. EPA, 2003. United States Environmental Protection Agency. Developing Relative Potency Factors for Pesticide Mixtures: Biostatistical Analyses of Joint Dose-Response. EPA/630/R-03/052. 